PSFC/JA-03-26

\title{
Initial Active MHD Spectroscopy Experiments Exciting Stable Alfvén Eigenmodes in Alcator C-Mod
}

\author{
J.A. Snipes, D. Schmittdiel, A. Fasoli*, R.S. Granetz, R.R. Parker
}

16 December 2003

Plasma Science and Fusion Center

Massachusetts Institute of Technology

Cambridge, MA 02139 USA

\author{
*CRPP-EPFL \\ Association EURATOM-Confédération Suisse \\ 1015 Lausanne, Switzerland
}

This work was supported by the U.S. Department of Energy, Cooperative Grant No.

DE-FC02-99ER54512. Reproduction, translation, publication, use and disposal, in whole or in part, by or for the United States government is permitted.

Submitted for publication to Plasma Physics and Controlled Fusion. 


\title{
Initial Active MHD Spectroscopy Experiments Exciting Stable
}

\section{Alfvén Eigenmodes in Alcator C-Mod}

\author{
J A Snipes, D Schmittdiel, A Fasoli, R S Granetz, R R Parker \\ MIT Plasma Science and Fusion Center, Cambridge, MA 02139 USA \\ *CRPP-EPFL, Association EURATOM-Confédération Suisse, 1015 Lausanne, Switzerland
}

\begin{abstract}
A pair of toroidally localised in-vessel antennas in Alcator C-Mod were used to excite and detect stable Toroidal Alfvén Eigenmode (TAE) resonances in the frequency range of $400-$ $500 \mathrm{kHz}$. By ramping the toroidal field, the plasma TAE frequency was swept through the constant antenna excitation frequency to find a resonance and the width of the resonance was measured to determine the effective damping rate of the mode. The toroidal field and density at the resonances were observed to scale as $B_{T} \propto n_{e}^{0.5}$, as expected for Alfvén eigenmodes. TAE damping rates were measured in both inner wall limited and diverted discharges. The inner wall limited damping rates for these discharges were in the range of $1 \%<|\gamma / \omega|<4 \%$. Diverted plasmas, on the other hand, required a very small outer gap between the last closed flux surface (LCFS) and the outboard limiter of less than $1 \mathrm{~cm}$ to observe the resonances and the damping rates were then very low with $|\gamma / \omega|<1 \%$.
\end{abstract}

\section{Introduction}

Moderate mode toroidal number $\mathrm{n} \sim 10$ Alfvén eigenmodes (AEs) are expected to be unstable [1] in the next step fusion device ITER [2], which will be dominated by fast $\alpha$ particle heating. If Alfvén eigenmodes are not sufficiently damped, they may lead to anomalous losses of the fast ions, which could quench the fusion burn or if the losses are highly localised could lead to rapid and substantial damage to the first wall [3]. It is therefore important to understand the physics of the damping of AEs and how it may scale to next step fusion devices.

The growth rate of AEs is determined by a balance of the fast ion drive and the damping due to a number of mechanisms such as continuum damping [4], radiative damping [5], and ion Landau damping [6]. By exciting modes with external antennas in Ohmic plasmas with no fast particle drive, it is possible to isolate the damping from the drive mechanisms. Previous work on JET [7-11] has extensively studied the damping rates of low $\mathrm{n}=0,1$, and 2 stable AEs excited with large saddle coils across a broad range of plasma conditions, where they have found a clear dependence of the damping, for example, on plasma shape and edge magnetic shear. The damping rate was found to increase with increased triangularity and elongation consistent with the increased edge magnetic shear. The damping rate was lowest with low elongation, low triangularity 

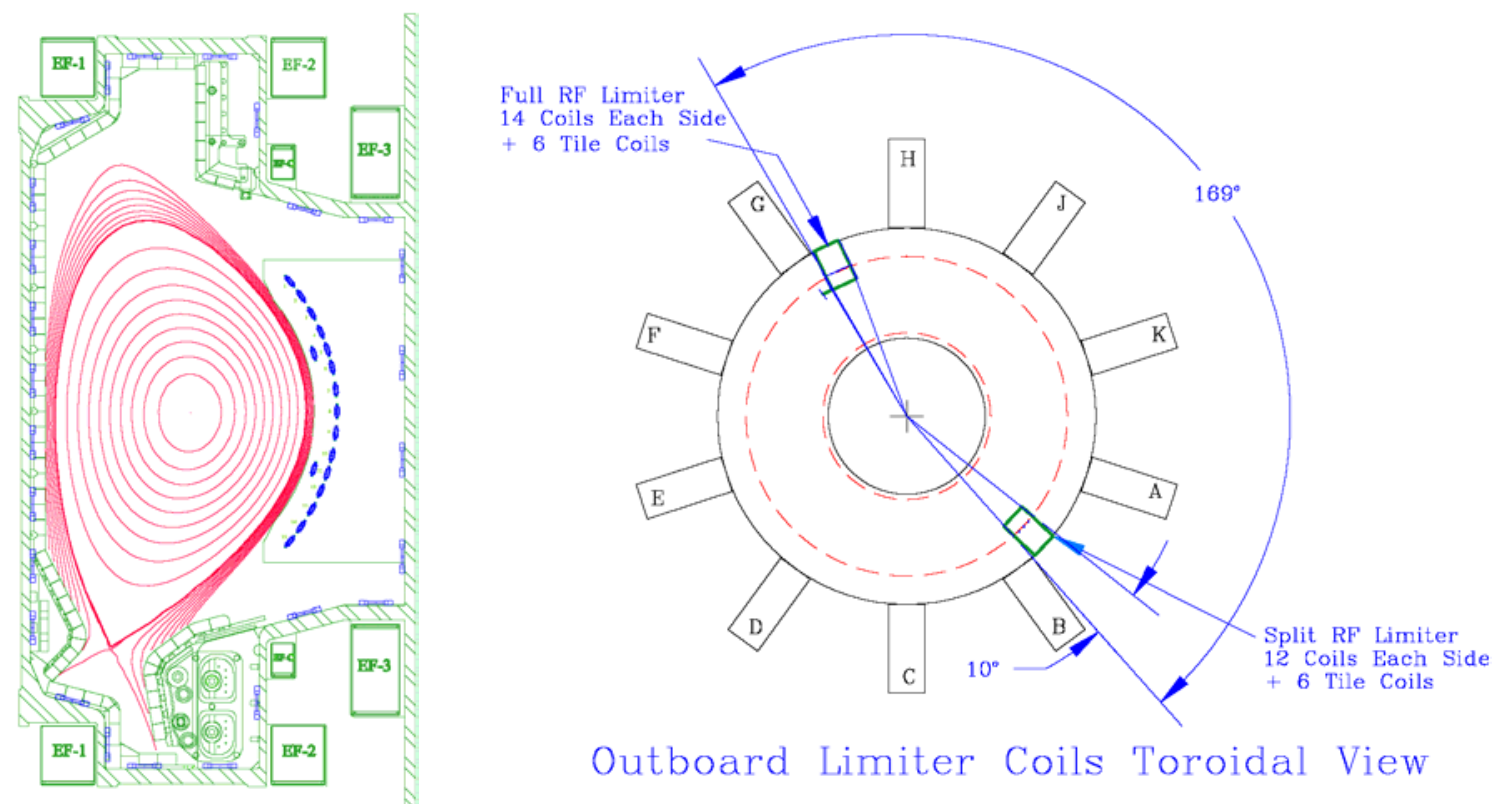

Outboard Limiter Coils Toroidal View

Figure 1. a) Poloidal cross-section of Alcator C-Mod showing the locations of the poloidal field pick-up coils (in blue) on one of the outboard limiters as well as the equilibrium pick-up coils along the wall of the vacuum vessel, b) a plan view of Alcator C-Mod showing the toroidal locations of the two outboard limiters on which the pick-up coils are mounted.

inner wall limited plasmas. Indeed, in diverted plasmas the damping rates exceeded $|\gamma / \omega|$ $>5-10 \%[12]$.

With the inspiration and close collaboration of the JET experiments, a pair of active MHD antennas was installed in Alcator C-Mod in 2002. In this way, the damping rates of AEs could be measured at the same densities and toroidal fields expected in ITER as well as with the moderate $\mathrm{n}$ mode numbers expected in ITER. This paper presents the initial results demonstrating that TAE resonances are clearly excited by a toroidally and poloidally localised antenna structure in both inner wall limited and diverted plasmas in C-Mod and documents the measured damping rates for these conditions.

\section{Active MHD Diagnostics}

MHD modes are measured with several arrays of poloidal field pick-up coils. Figure 1 shows both a poloidal cross-section of Alcator C-Mod with the locations of the magnetic pick-up coils on one of the two outboard limiters as well as a plan view showing the toroidal locations of the two outboard limiters. There are a total of 65 poloidal field pick- 

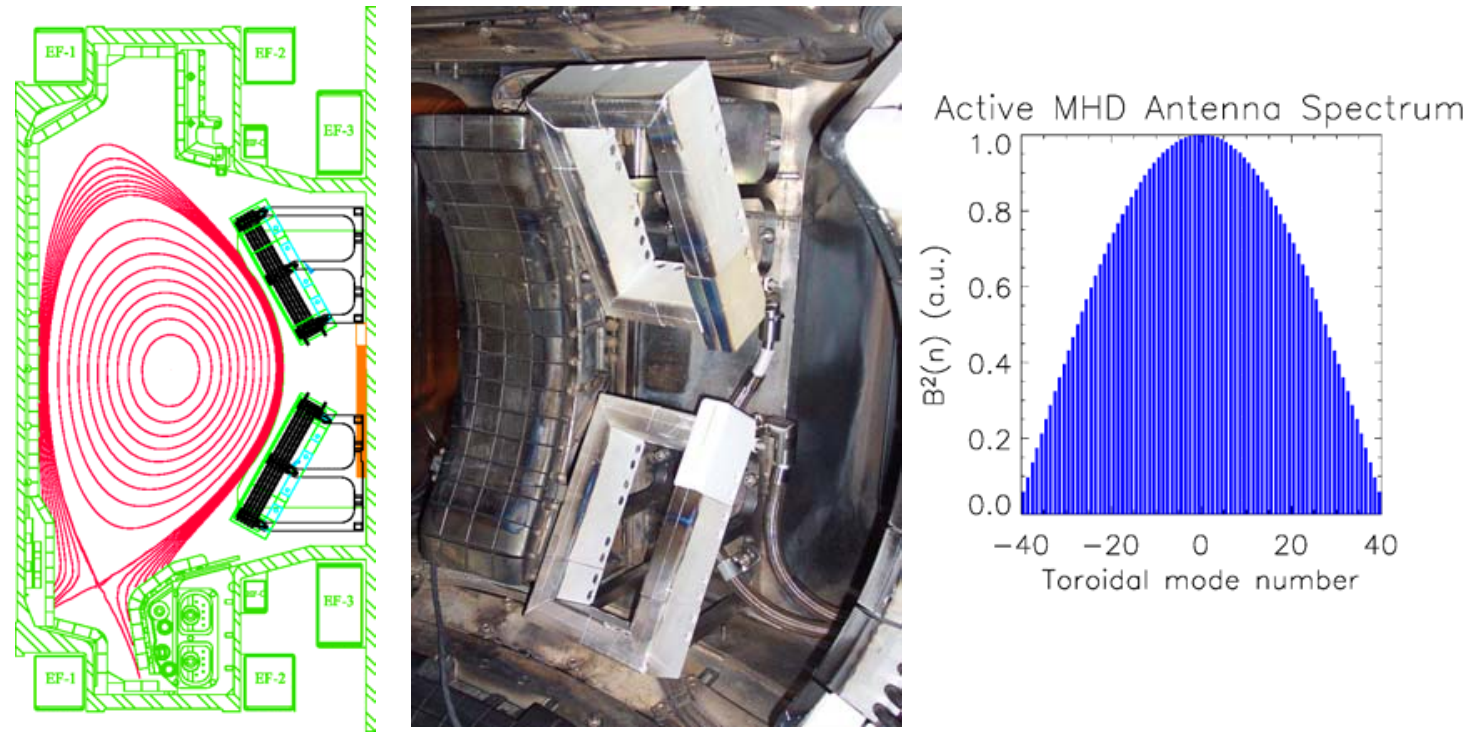

Figure 2. a) Poloidal cross-section of Alcator C-Mod showing the locations of the two active MHD antennas relative to the outboard limiter and a typical plasma equilibrium, b) Photo of the active MHD antennas in C-Mod c) Calculated toroidal mode spectrum of the active MHD antennas with a FWHM of $\mathrm{n} \sim 20$.

up coils mounted to the two limiters. There are up to 15 coils in a poloidal array on one of the limiters allowing estimates of poloidal mode numbers up to $\mathrm{m}<14$ to be determined. In addition to the coils mounted to the sides of the limiters, there are two sets of three pick-up coils mounted beneath the molybdenum tiles at a slit between the tiles in each limiter at $\pm 10 \mathrm{~cm}$ above and below the midplane that are separated by $2.4^{\circ}$ toroidally. These allow toroidal mode numbers up to $\mathrm{n}<75$ to be measured. The pickup coil signals are digitised at up to $2.44 \mathrm{MHz}$ sampling rate throughout most of the discharge.

Alfvén eigenmodes are excited with a pair of active MHD antennas at one toroidal location. Figure 2 shows a poloidal cross-section of Alcator C-Mod with the location of the two antennas shown relative to the outboard limiter and a typical plasma equilibrium as well as a photo of the antennas and limiter and a calculation of the toroidal mode spectrum of the antennas. Each antenna consists of five turns that are $15 \times 25 \mathrm{~cm}$ in area. The stainless steel windings are protected from the plasma by insulating boron nitride tiles, which allow the last turn closest to the plasma to be only $2.5 \mathrm{~cm}$ from the limiter radius. The windings are secured to the antenna frame with ceramic coated stainless steel bolts and the turns are insulated from one another and the frame with 
ceramic coated stainless steel washers and mica washers for added high voltage standoff. These are particularly important to avoid RF breakdown across the antenna because of the strong coupling to the $4 \mathrm{MW}$ ICRF antenna located $\sim 40 \mathrm{~cm}$ away (just visible in the lower right hand side of the photo). The antennas are connected to $15 \mathrm{kV} / 15 \mathrm{~A}$ coaxial feedthroughs via ceramic beaded coaxial transmission lines. A geometrical calculation of the toroidal mode spectrum of each antenna based indicates that they excite a broad range of toroidal mode numbers as shown in Figure 2 with a FWHM of $|n|<20$. In the frequency range of interest $(<1 \mathrm{MHz})$ each antenna remains inductive with a resistance of $\sim 1 \Omega$ and an inductance of $\sim 9 \mu \mathrm{H}$. One side of each antenna is grounded to the vacuum vessel at the coaxial vacuum feedthrough. Just on the air side of the feedthrough, each antenna is connected to $10 \mathrm{~kW}$ low-pass RF filters that attenuate the ICRF frequencies $(50-80 \mathrm{MHz})$ by up to $-90 \mathrm{~dB}$ while leaving frequencies below 1 MHz essentially unaffected in both amplitude and phase.

For the results of this paper, only the upper antenna was excited first by an ENI AP400B $400 \mathrm{~W}$ amplifier, which when properly matched to the antenna, could drive up to $12 \mathrm{~A}$ of current across a frequency range from $80 \mathrm{kHz}$ to $2.7 \mathrm{MHz}$. Later this amplifier was replaced by an MIT designed amplifier that, when properly matched, could excite the antenna with up to $12 \mathrm{~A}$ across a frequency range from a few $\mathrm{kHz}$ to nearly $1 \mathrm{MHz}$. For these initial results, the matching network consisted of parallel and series capacitances tuned to a particular frequency with a FWHM of only $\pm 50 \mathrm{kHz}$ around the peak frequency. The narrow bandwidth of the matching circuit meant that the plasma resonances were most easily observed when exciting the antenna at a constant frequency and that the frequency could not be swept over a broad range to search for a resonance.

The calculated radial field perturbation produced by one active MHD antenna driven with 10 A of current is $\tilde{B}_{r} \sim 5 \times 10^{-5} \mathrm{~T}$ at a distance of $12 \mathrm{~cm}$ from the antenna or $\mathrm{r} / \mathrm{a} \sim 0.5$. So, the perturbations produced by the active MHD antenna can only be observed by rather sensitive magnetic diagnostics and do not otherwise perturb the plasma equilibrium. 

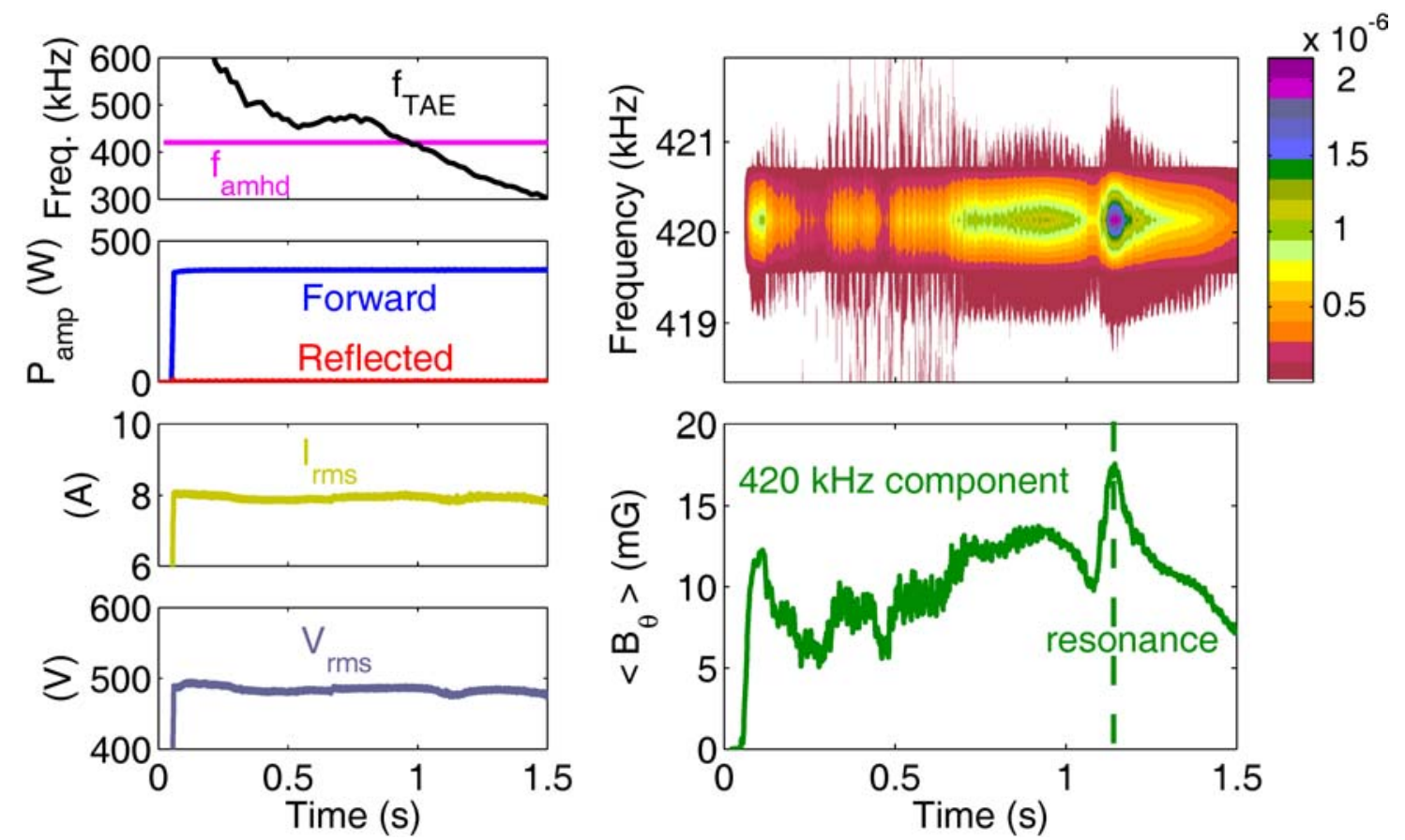

Figure 3. Fourier analysis spectrogram of a typical TAE resonance in C-Mod with the active MHD antenna excited at a constant frequency of $420 \mathrm{kHz}$ and the plasma TAE frequency swept across the antenna frequency by ramping the toroidal field from 6.5 to $4 \mathrm{~T}$. The resonance is clearly visible in both the spectrogram and the average poloidal field fluctuation vs time just as the plasma center of the TAE gap frequency crosses the active MHD frequency. The RMS current and voltage across the antenna are also shown together with the forward and nearly zero reflected power.

\section{Initial Alfvén Eigenmode Experiments}

The objectives of these initial active MHD experiments in C-Mod were to demonstrate that it is possible to excite stable resonant Alfvén eigenmodes with a localised intermediate $\mathrm{n}$ antenna and to measure the damping rate of these modes. Observable resonances in the pick-up coil signals are expected when the excitation frequency of the active MHD system approaches a resonant TAE frequency in the plasma. The center of the TAE frequency gap is given approximately by $f_{T A E}=\frac{v_{A}}{4 \pi q R} \propto \frac{B_{T}}{q R \sqrt{n_{e} m_{i}}}$. So, the first check to perform experimentally was to vary the plasma density and toroidal field and determine how the resonant frequencies scaled with these parameters.

Theoretically, the least stable TAE is expected to be driven at or near the $\mathrm{q}=1.5$ surface [13]. For low $n$ stable TAEs excited in monotonic shear plasmas in JET, the resonant q value was found to be approximately 1.5 [12]. So, based on the JET experience, the initial C-Mod experiments were setup to excite frequencies centered 


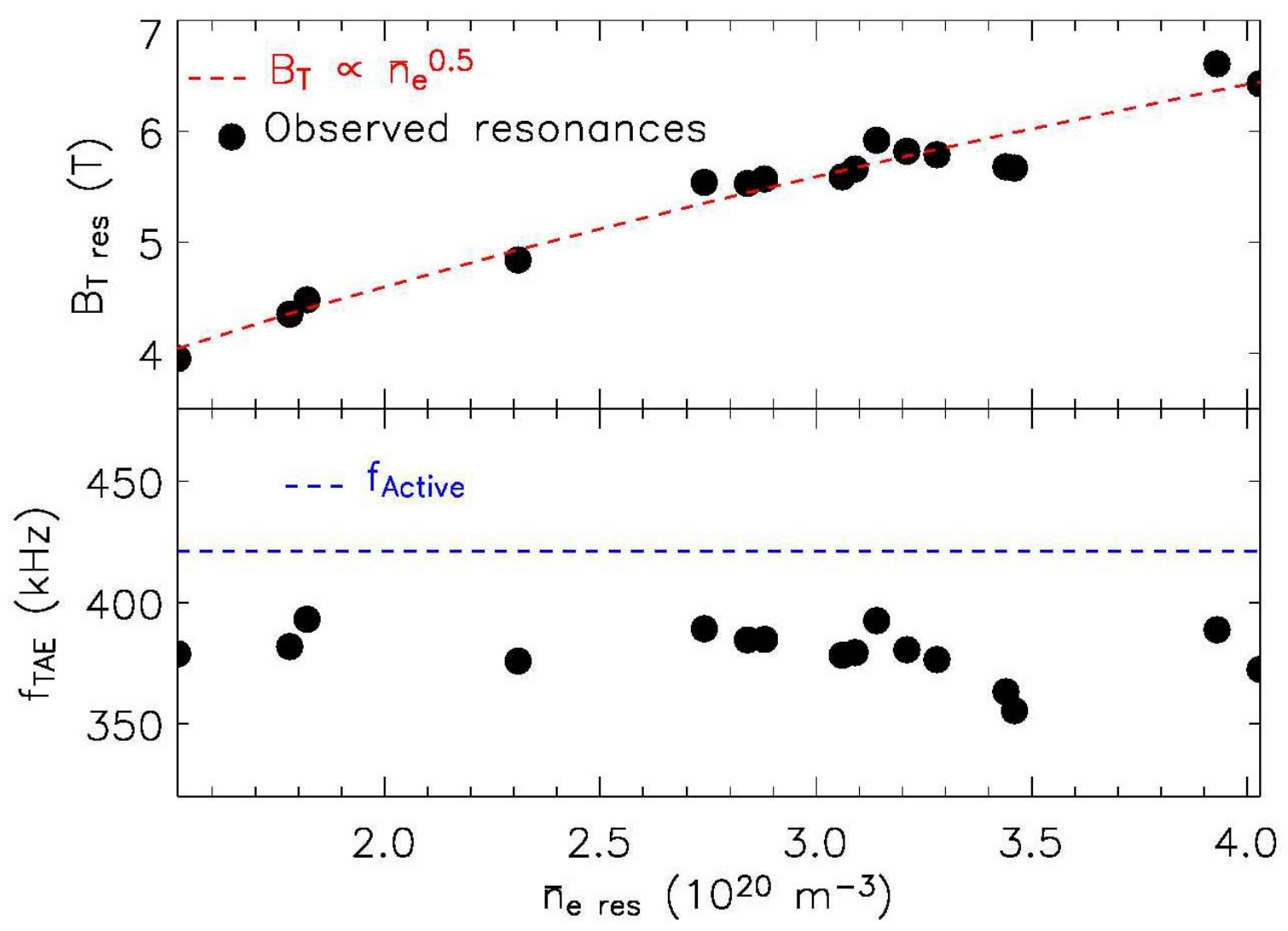

Figure 4. The scaling of the toroidal field and line averaged electron density at the times of the observed mode resonances for a series of discharges with constant active MHD excitation frequency at $420 \mathrm{kHz}$. The toroidal field increases with the square root of the density as expected for Alfvén eigenmodes. The calculated center of the TAE gap frequency at the resonances lies within $10-15 \%$ of the excited active MHD frequency indicating that these resonances are indeed TAEs.

about the TAE gap at $\mathrm{q}=1.5$ in low elongation $(\kappa=1.3)$ inner wall limited plasmas, where the damping rates were observed to be lower in JET. Because of the limited bandwidth of the matching circuit, rather than sweeping the antenna frequency, we chose to excite the antenna at a constant frequency of $420 \mathrm{kHz}$, where we could precisely tune the matching circuit and then ramp the toroidal field in time at constant density to scan the TAE frequency in the plasma. Figure 3 shows a typical TAE resonance in C-Mod that is clearly visible in both the Fourier spectrogram of a magnetic pick-up coil signal as well as in the average poloidal field fluctuation amplitude versus time just as the plasma TAE frequency crosses the active MHD antenna excitation frequency.

By maintaining the same toroidal field ramp and then varying the flattop electron density from shot to shot, the scaling of the observed mode resonant frequencies with 


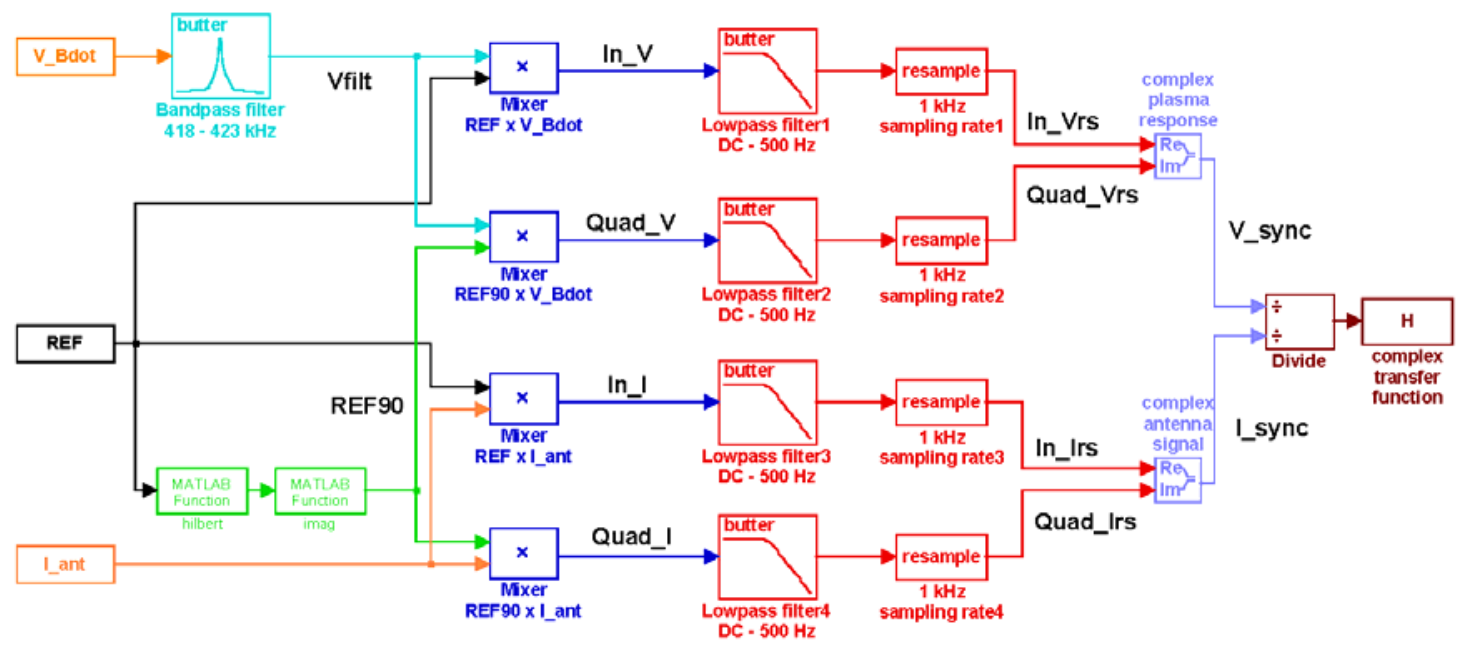

Figure 5. A schematic of the software synchronous detection algorithm used to analyse active MHD mode resonances in C-Mod.

toroidal field and density could be determined and compared with that expected for TAEs. Figure 4 shows how the toroidal field scales with the line averaged electron density at the time of the observed resonances across this series of discharges. The square root dependence of the toroidal field on electron density is as expected for Alfvén eigenmodes. The calculated center of the TAE gap frequency at $\mathrm{q}=1.5$ lies within $10-$ $15 \%$ of the excited active MHD frequency, which is well within the TAE gap, indicating that these are indeed TAE resonances.

\section{Software Synchronous Detection}

Because the observed mode resonances have small amplitude $\left(\tilde{B}_{\theta} \sim 10^{-6} \mathrm{~T}\right)$, a synchronous detection technique is employed using data from multiple pick-up coils to more clearly identify the mode resonances above the background noise level. While such synchronous detection was performed with hardware electronic circuits on JET [14], we chose to fast sample the data and perform synchronous detection with software to allow more flexibility in the analysis of the data.

The software synchronous detection algorithm is outlined in Figure 5. The initial narrow band pass filters the data typically within $5 \mathrm{kHz}$ of the excitation frequency and the mixed components are then low pass filtered to $500 \mathrm{~Hz}$ to distinguish the slowly 
varying amplitude and phase of the resonance. The ratio of the synchronously detected pick-up coil signal to the antenna current then gives the complex transfer function.

The transfer function can be modeled as a rational fraction of complex polynomials in the frequency domain with a resonance as a pole [15]:

$$
H(\omega, x)=\frac{V_{\text {sync }}}{I_{\text {sync }}}=\frac{1}{2}\left[\frac{r(x)}{i \omega-p}+\frac{r^{*}(x)}{i \omega-p^{*}}\right]+D(\omega, x)=\frac{B(\omega, x)}{A(\omega)} .
$$

Here $\mathrm{x}$ refers to the different coil positions. By comparing toroidally separated coils, the phase of the residue $r$ can be used to determine the toroidal mode number. The quantity $p=i \omega_{0}+\gamma$ is a pole in the complex plane so that the normalized damping rate of the mode is then $\gamma / \omega_{0}$. The expression $D(\omega, x)$ represents the unavoidable direct coupling of the active MHD antenna to the pick-up coil. Since the resonance should have the same damping rate on all pick-up coil signals, a single pole is specified for all coils while the residues depend on each coil. By analyzing multiple coils simultaneously, statistical errors can be reduced for determining the damping rate of the mode. Then, the fit to a ratio of polynomials in $\omega$ has the same denominator $A(\omega)$ with order nA while the numerator $B(\omega, x)$ has order $\mathrm{nB}$ and is specific for each coil. Typically, $\mathrm{nA}=2$ while $\mathrm{nB}$ $=5$ or 6 will adequately fit the data. A clear resonance in the transfer function should sweep out a circle in the complex plane as the plasma parameters are changed in time to change the TAE frequency in the plasma.

\section{Stable TAE Resonances in Inner Wall Limited Plasmas}

Figure 6 shows an example of a resonance and the fit to the transfer function of a pick-up coil in the complex plane for an inner wall limited plasma. In this case, the fit determined the damping rate to be $|\gamma / \omega| \sim 3 \%$. Stable TAE damping rates have been measured for inner wall limited plasmas on C-Mod to be in the range of $1.4 \%<|\gamma / \omega|<$ $4 \%$, which is in the same range as the damping rates observed in JET inner wall limited plasmas [9].

Given the broad toroidal mode spectrum with FWHM $n<20$ of the active MHD antenna (Figure 2), it is not clear what determines with which toroidal mode number the plasma responds. By comparing the phase of the residues of the transfer functions of 

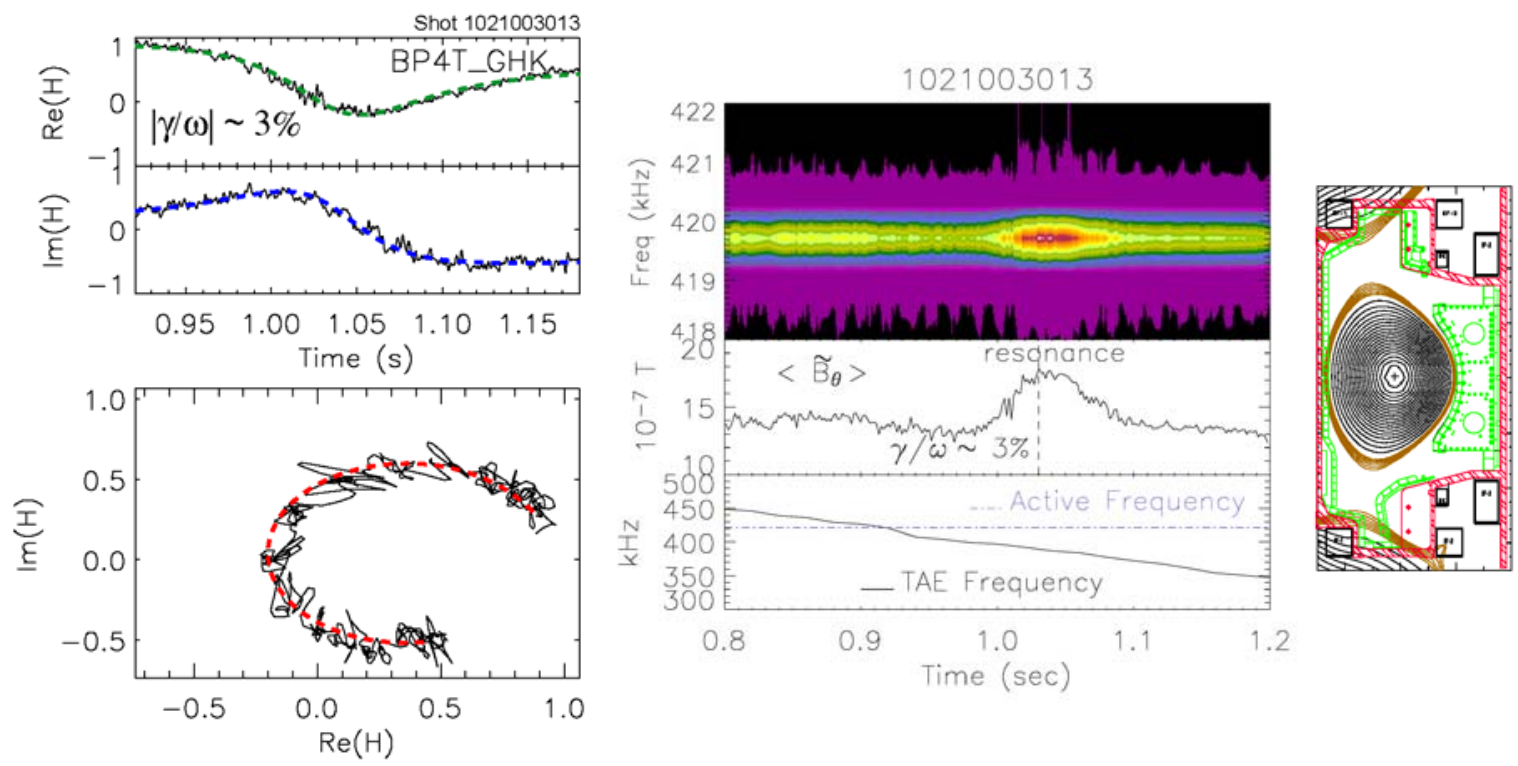

Figure 6. A TAE resonance in an inner wall limited plasma together with the fit to the transfer function in the complex plane. The fit determines the damping rate $|\gamma / \omega| \sim 3 \%$ for this resonance. While the resonance does not occur exactly when the plasma TAE center of the gap frequency crosses the active MHD frequency, it still lies well within the TAE gap.

toroidally separated pick-up coils, the toroidal mode number of the resonant mode was found to be $n=4$ for the resonance shown in Figure 6, though a more systematic analysis of toroidal mode numbers remains to be done. Such moderate toroidal mode numbers are closer to the TAE mode numbers that are expected to be unstable in ITER [1] than the low $\mathrm{n}=0,1$, and 2 modes that have been excited with the saddle coils in JET. New TAE antennas are presently under construction for JET that will have a toroidal mode spectrum up to $\mathrm{n}=10$ to excite the mode numbers expected in ITER [10].

\section{Stable TAE Resonances in Diverted Plasmas}

Actively excited stable TAE resonances were also observed in diverted plasmas on CMod when the outer gap between the last closed flux surface and the limiter radius was less than about $1 \mathrm{~cm}$. Figure 7 shows an example of three TAE resonances being excited as the plasma TAE frequency oscillates through the constant active MHD antenna frequency due in this case, in addition to the toroidal field ramp, to a change in the plasma density. The mode damping rate was found to be $|\gamma / \omega| \sim 1 \%$, significantly lower than observed at JET $[11,12]$, which for low n numbers in diverted plasmas with high edge shear have damping rates exceeding $5-10 \%$. 


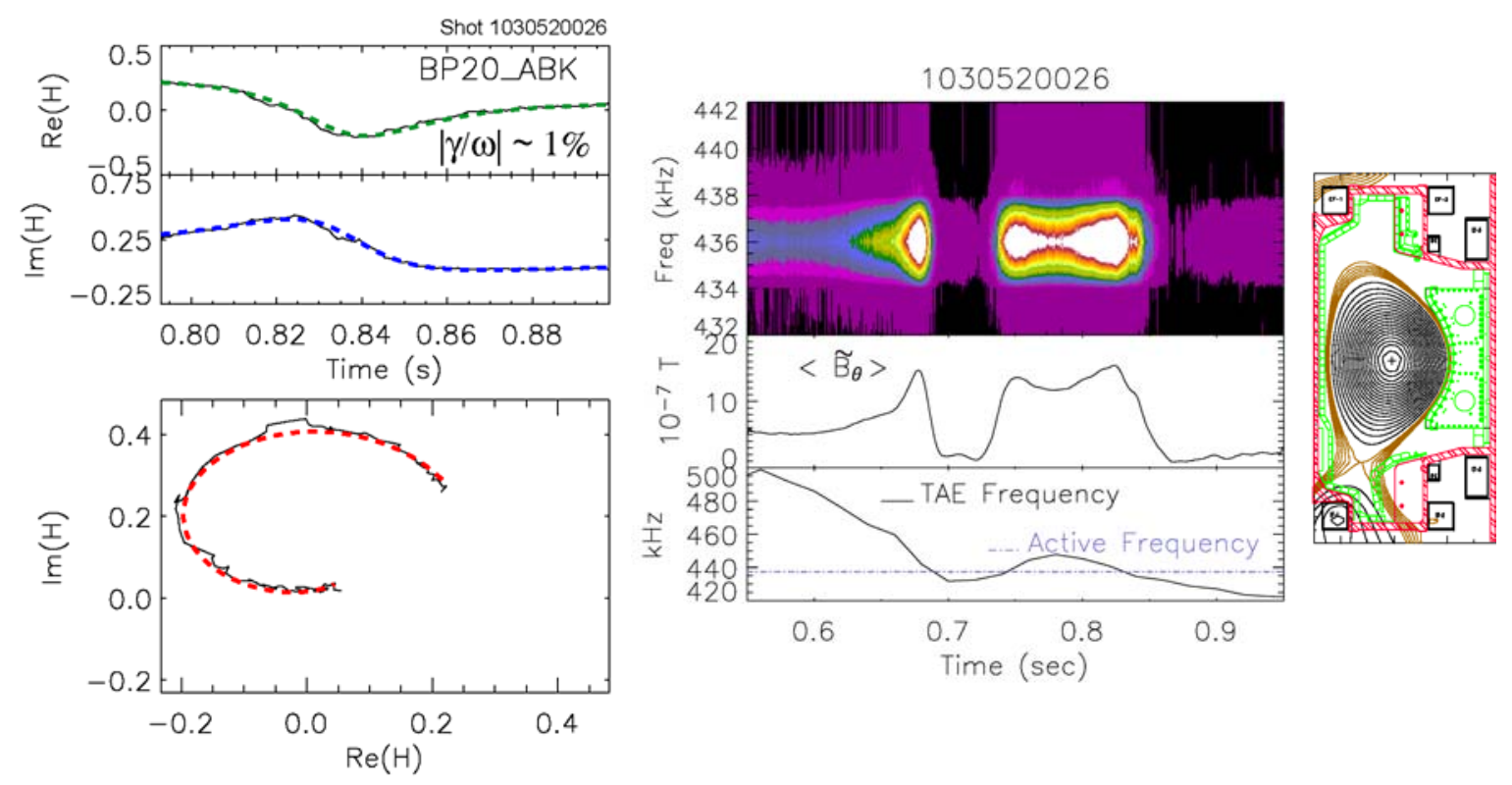

Figure 7. Three stable TAE resonances observed in a diverted plasma as the plasma TAE frequency oscillates through the constant active MHD frequency three times. The fit of the transfer function for the third resonance is shown on the left of the figure in the complex plane. The damping rate was found to be particularly low compared to inner wall limited plasmas with $|\gamma / \omega| \sim 1 \%$.

To investigate this effect further, an experiment was devoted to comparing the measured damping rate of stable TAEs for inner wall limited and diverted plasmas as a function of changes in the outer gap. Figure 8 shows the measured TAE damping rate as a function of outer gap for limited and diverted plasmas. The results were particularly unexpected in that for small outer gaps $<1 \mathrm{~cm}$ in limited plasmas, resonances were no longer observed. The damping rate of limited plasmas increased with increasing outer gap between 1 and $2.5 \mathrm{~cm}$. For diverted plasmas, on the other hand, only when the outer gap was $<1 \mathrm{~cm}$ were resonances observed and they had very low damping rates between $0.4 \%<|\gamma / \omega|<1.4 \%$ that appear to decrease with decreasing outer gap. For outer gaps larger than about $1 \mathrm{~cm}$, resonances were no longer observed in diverted plasmas. The lack of observed resonances under these conditions may be due to reduced coupling to the mode or exceedingly high damping rates.

\section{Conclusions and Future Work}

The initial active MHD experiments exciting only one antenna on C-Mod have demonstrated that stable TAE resonances can be excited and observed in both inner wall 


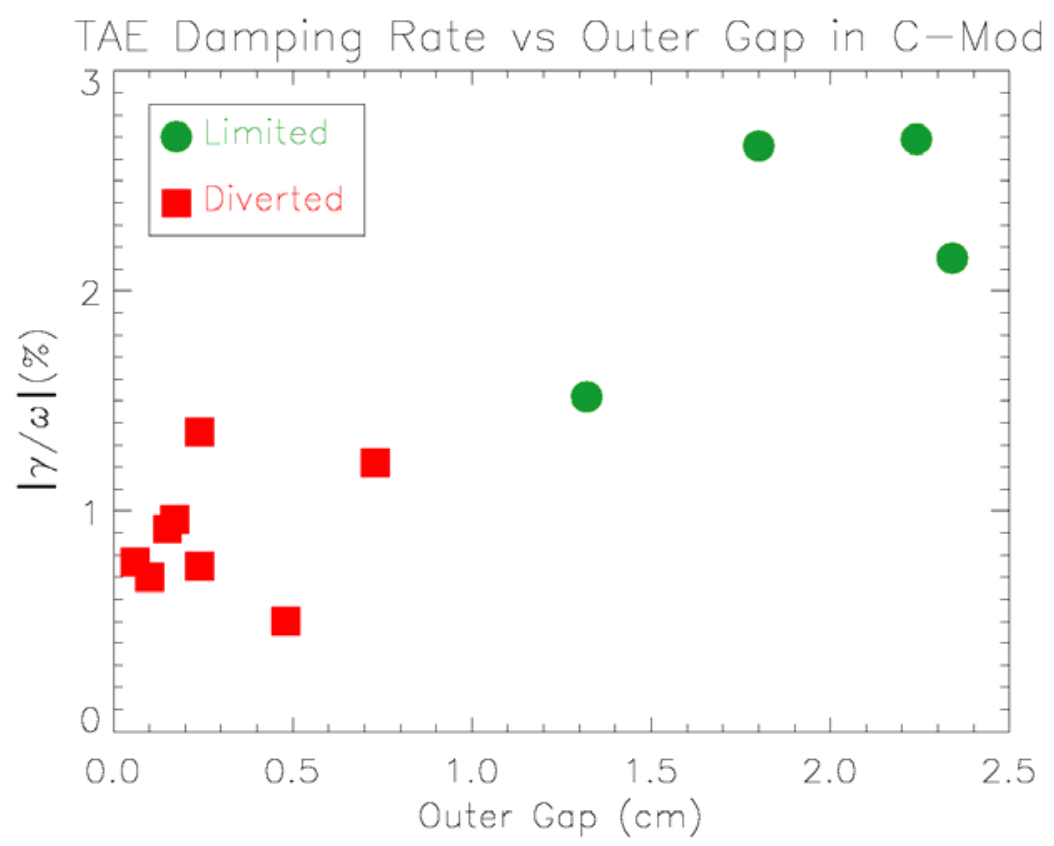

Figure 8. Stable TAE damping rate as a function of outer gap for both inner wall limited and diverted plasmas in C-Mod. For outer gaps $<1 \mathrm{~cm}$, resonances were not observed in limited plasmas whereas for outer gaps $>1 \mathrm{~cm}$, resonances were not observed in diverted plasmas.

limited and diverted plasmas in the range of densities and toroidal fields expected in ITER. The resonances were excited at a constant active MHD antenna frequency for ramping toroidal field at various flattop densities. The toroidal field at resonance scaled as the square root of the line averaged density as expected for Alfvén eigenmodes. The resonances are consistent with a center of the TAE gap frequency corresponding to a $\mathrm{q}$ value near 1.5.

A software synchronous detection routine provides good fits to the stable TAE resonances on multiple pick-up coil signals. The fitted transfer function in the complex plane yields damping rates between $1.4 \%<|\gamma / \omega|<4 \%$ for inner wall limited plasmas in agreement with JET results. For diverted plasmas, on the other hand, the resonances were only observed when there were particularly small outer gaps of $<1 \mathrm{~cm}$. In these diverted cases, the damping rates were much lower in the range $0.4 \%<|\gamma / \omega|<1.4 \%$.

The active MHD amplifier and power supply on C-Mod are presently being upgraded to excite both antennas with up to $20 \mathrm{~A}$ of current simultaneously [18]. The matching circuit will automatically switch capacitors to maintain matching to the antenna 
as the frequency sweeps across a broad range. A new digital control computer for C-Mod plasma operation will provide real time feedback control on the TAE frequency.

\section{Acknowledgements}

The authors would like to thank R. Vieira and W. Burke for their expert engineering assistance in designing and building the active MHD antennas and amplifiers. We would also like to thank the Alcator C-Mod team without whose help these results would not have been possible. This work was supported by DoE grant DE-FC02-99ER54512.

\section{References}

[1] Gorelenkov N N et al 2003 Nucl Fus 43594

[2] Aymar R et al 2001 Nucl Fus 411301

[3] Wong K-L 1999 Plasma Phys Cont Fus 41 R1

[4] Zonca F and Chen L 1992 Phys Rev Lett 68592

[5] Mett R R and Mahajan S M 1992 Phys Fluids B 42885

[6] Betti R and Freidberg J P 1992 Phys Fluids B 41465

[7] Fasoli A et al 1995 Phys Rev Lett 75645

[8] Fasoli A et al 1997 Plasma Phys Cont Fus 39 B287

[9] Fasoli A et al 2000 Phys. Plasmas 71816

[10] Fasoli A et al 2002 Plasma Phys Cont Fus 44 B159

[11] Testa D et al 2001 Nucl Fus 41809

[12] Testa D et al 2003 Nucl Fus 43479

[13] Poedts S et al 1992 Plasma Phys Cont Fus 341397

[14] Fasoli A et al 1995 Nucl Fus 351485

[15] Moret J-M 1994 CRPP Report LRP 498/94

[16] Snipes J A et al 1999 Proc 26 $6^{\text {th }}$ EPS Conference, Maastricht 23J 293

[17] Snipes J A et al 2000 Plasma Phys Cont Fus 4238

[18] W. Burke et al 2003 "Broadband Amplifiers for the Active MHD Diagnostic on Alcator C-Mod," submitted for publication in Proceedings of the 20th IEEE/NPSS Symposium on Fusion Engineering (SOFE) 